\title{
Finite element analysis of the LOLA receiver telescope lens
}

\author{
Elizabeth A. Matzinger
}

NASA Goddard Space Flight Center, Greenbelt, MD, USA 20740

\begin{abstract}
This paper presents the finite element stress and distortion analysis completed on the receiver telescope lens of the Lunar Orbiter Laser Altimeter (LOLA). LOLA is one of six instruments on the Lunar Reconnaissance Orbiter (LRO), scheduled to launch in 2008. LOLA's main objective is to produce a high-resolution global lunar topographic model to aid in safe landings and enhance surface mobility in future exploration missions. A receiver telescope captures the laser pulses transmitted through a diffractive optical element (DOE) and reflected off the lunar surface. The largest lens of the receiver telescope was modeled with solid elements and constrained in a manner consistent with the behavior of the mounting configuration. Twenty-one temperature load cases were mapped to the nodes based on thermal analysis completed by LOLA's lead thermal analyst, and loads were applied to simulate the preload applied from the ring flexure. The thermal environment of the baseline design produces large radial and axial gradients in the lens. These large gradients create internal stresses that may lead to part failure, as well as significant bending that degrades optical performance. The high stresses and large distortions shown in the analysis precipitated a design change from BK7 glass to sapphire.
\end{abstract}

Keywords: LRO, LOLA, Finite Element Analysis, BK7 glass

\section{INTRODUCTION}

The Lunar Orbiter Laser Altimeter (LOLA) is an instrument selected as one of six instruments on the Lunar Reconnaissance Orbiter (LRO). LRO, scheduled to fly to the Moon in 2008, is the first step in NASA's Lunar Precursor Robotic Program (LPRP), and has a mission objective to conduct investigations that will be specifically targeted to prepare for and support future human exploration of the Moon. LOLA will assist in this endeavor by producing a highresolution global lunar topographic map and measure landing site slopes that will enable precise targeting, safe landing, and surface mobility to carry out exploratory activities. LOLA will also search for lunar surface ice inside permanently shadowed craters near the poles.

LOLA, shown in Figure 1, is a Goddard Space Flight Center (GSFC) instrument that has direct heritage from a previous GSFC instrument, the Mercury Laser Altimeter (MLA), currently en route to Mercury onboard NASA's MESSENGER spacecraft. The LOLA laser transmits pulses that are split into five beams by a diffractive optical element (DOE). The receiver telescope captures the beams reflected off the lunar surface and focuses them into a fiber optic array at the telescope focal plane. The receiver, a $14 \mathrm{~cm}$ diameter telescope with an effective focal length of $500 \mathrm{~mm}$, is made from beryllium to keep the instrument mass low and to minimize the thermal fluctuations expected on orbit. Lens 1 of the receiver telescope, labeled as "L1" in Figure 2, is subjected to large temperature swings and thus was analyzed by finite element analysis to determine the effect of the thermal environment on lens deformation and stress.

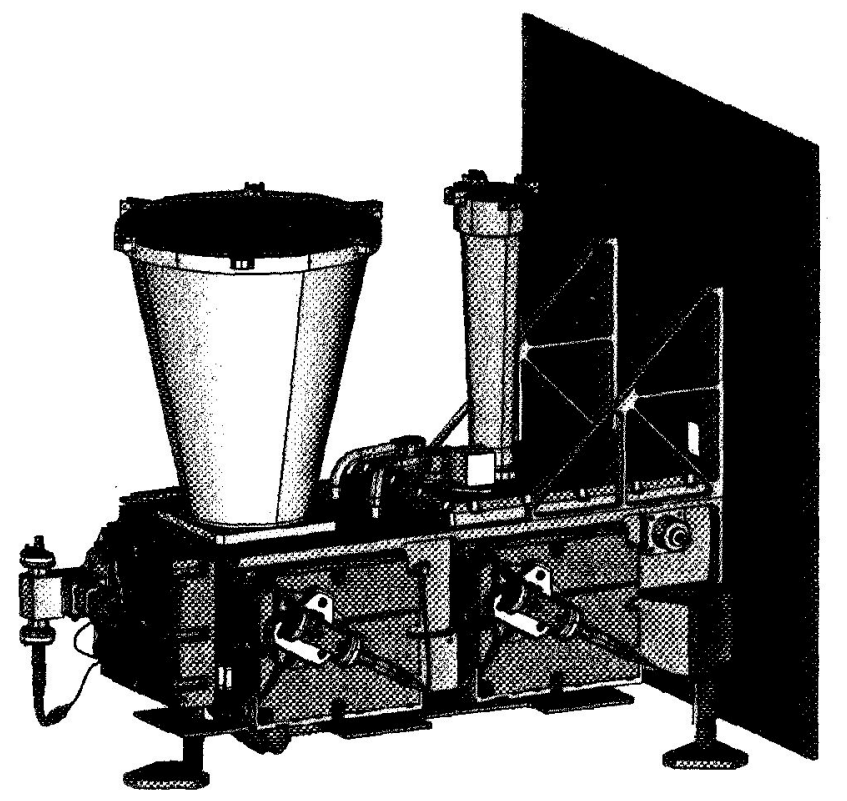

Fig. 1. LOLA Optical Transceiver Assembly 


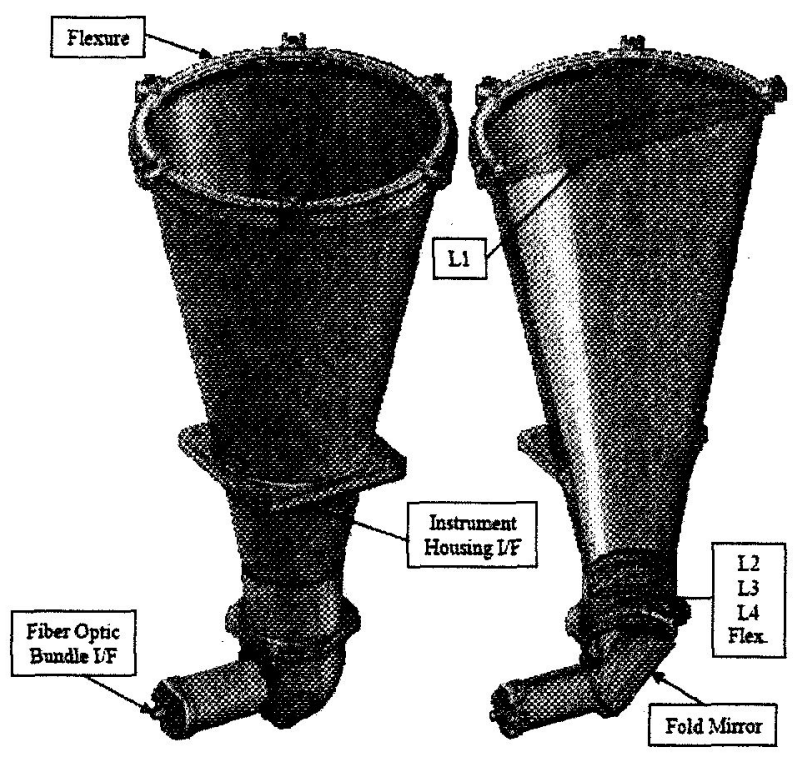

Fig. 2. LOLA Receiver Telescope

\section{MODEL DESCRIPTION}

FEMAP was the pre- and post-processor used to create the finite element model (FEM) of the receiver telescope lens 1 and view the analysis results. The finite element analysis (FEA) solver used was MSC.Nastran Version 2005. The FEM was created using 8-node CHEXA and 6-node CPENTA solid elements and consists of 9,305 nodes and 7,440 elements.

\subsection{Geometry and Constraints}

The LOLA receiver telescope lens analyzed is a $150 \mathrm{~mm}$ diameter aspheric lens. The shape of the lens was obtained from the CAD model and imported into FEMAP via a STEP file to facilitate meshing. The lens is meshed with four elements through the thickness in order to obtain the axial gradients discussed in section 2.3.2.

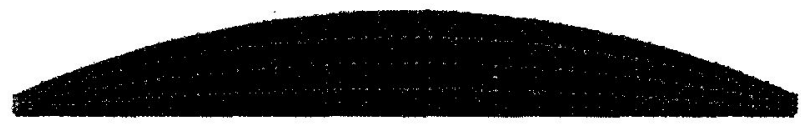

$+$

Fig. 3. Lens FEM cut-away side view

The FEM was constrained in a manner consistent with the behavior of the receiver telescope tube's mounting configuration, shown in Figure 4 . The lens was kinematically constrained at the center to allow for free radial expansion. In addition, the bottom edge of the lens was constrained in the $Z$ direction where the lens sits on the shelf of the receiver tube. 


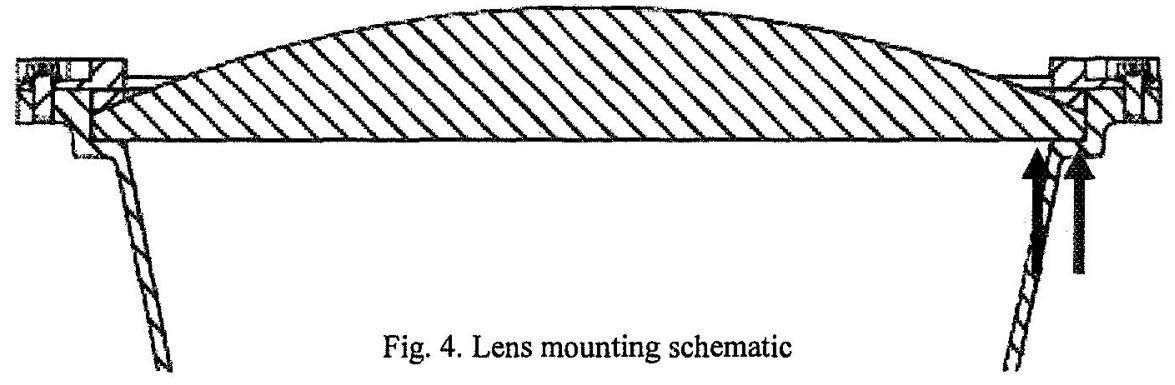

Constraining the bottom edge of the lens for the entire width of the shelf proved to be overly conservative, yielding unreasonably high stresses at the mounting locations. Thus, two separate constraint sets were created: one for the "hot" cases, where the lens would expand, and another for the "cold" cases. The hot case constraint fixed a ring of nodes in one degree of freedom - the $\mathrm{Z}$ direction - on the bottom outer edge of the lens. The cold case constraint moved this ring inward at the location of the inside edge of the shelf. These constraints allowed the lens to bend and pivot about one edge of the shelf instead of being clamped along the entire width of the shelf.

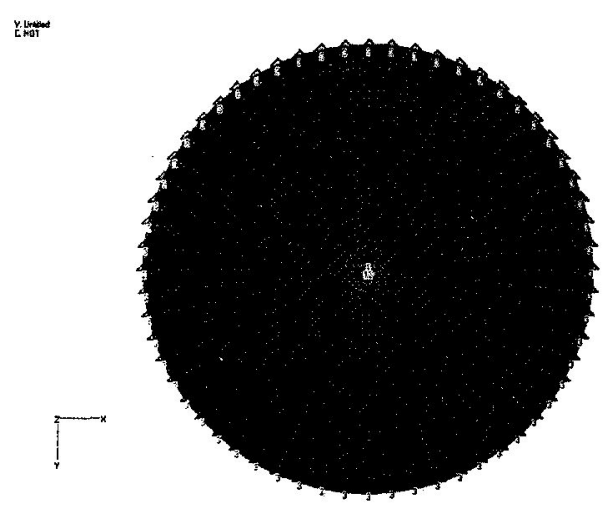

Fig. 5. Hot case constraints

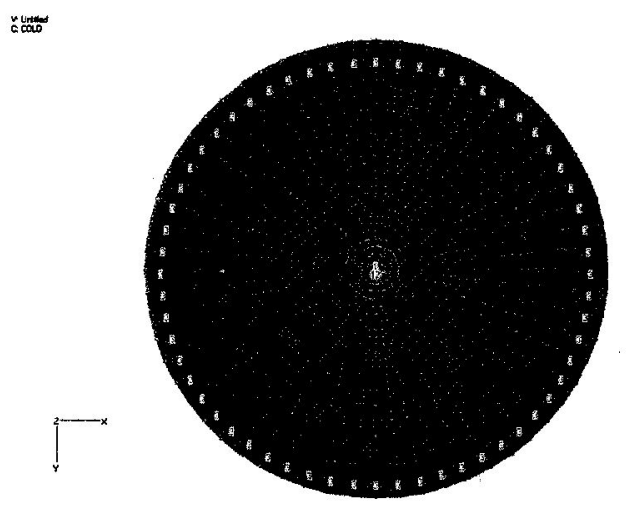

Fig. 6. Cold case constraints

\subsection{Material Properties}

The material properties for BK7 glass used for this analysis are listed in Table 1 below.

Table 1

\begin{tabular}{|c|c|}
\hline Young's Modulus, $\mathrm{E}$ & $8.067 \times 10^{4} \mathrm{MPa}\left(11.7 \times 10^{6} \mathrm{psi}\right)$ \\
\hline Poisson's ratio, $v$ & 0.208 \\
\hline $\begin{array}{c}\text { Coefficient of Thermal } \\
\text { Expansion (CTE), } \alpha\end{array}$ & $7.1 \mu \mathrm{m} / \mathrm{m}-{ }^{\circ} \mathrm{C}$ \\
\hline Density, $\rho$ & $2.77 \times 10^{-9} \mathrm{Mg} / \mathrm{mm} 3\left(0.1 \mathrm{lbs} / \mathrm{in}^{3}\right)$ \\
\hline Tensile Strength & $6.9 \mathrm{MPa}(1,000 \mathrm{psi})$ \\
\hline Compressive Strength & $34.5 \mathrm{MPa}(5,000 \mathrm{psi})$ \\
\hline
\end{tabular}

The tensile and compressive strengths are common "Rules of Thumb" for optical glass strength. Additional probabilistic analysis was completed by the GSFC Systems Safety and Reliability Office based on the lens surface finish and the surface area under tensile stress. 


\subsection{Applied Loads}

Twenty-one temperature load cases were mapped to the nodes based on thermal analysis completed by LOLA's lead thermal analyst. In addition, point loads were applied to simulate the preload applied from the ring flexure.

\subsubsection{Thermal Loads}

The thermal environment of the baseline design (an uncoated BK7 lens with no baffle) produces large radial and axial gradients in the lens. Two cases were run by the thermal analyst: a Beta $90^{\circ}$ cold operational case and Beta $0^{\circ}$ hot operational case. Figures 7 and 8 show the spacecraft orientation angle for both of these cases. The cold operational case is a steady state case, while the hot operational case is a transient case. The elapsed time of the hot case analysis covers three full orbits of the moon and consists of 268 time steps. A plot of the temperatures at each node of the thermal model for this transient case is shown in Figure 9. To attempt to bound the transient case, four time steps were chosen with the largest radial and axial gradients, both positive and negative. The resulting analysis did not convincingly show that these four cases would in fact be the bounding cases, so 14 additional cases were chosen at equal time spacing during the second orbit, between the $\mathbf{8 1 . 2}$ minute and 195.0 minute timesteps. As is shown in Section 3, several of the additional 14 temperature cases did result in higher stresses than the initial four cases chosen.

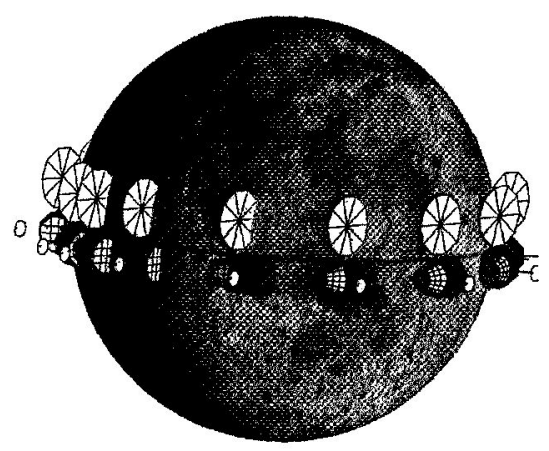

Fig. 7. Beta $0^{\circ}$

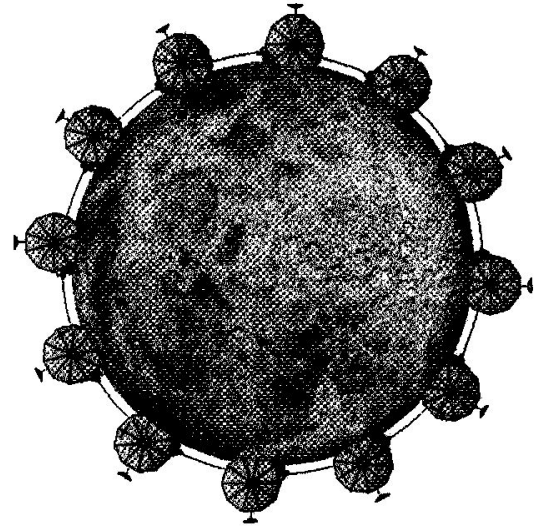

Fig. 8. Beta $90^{\circ}$

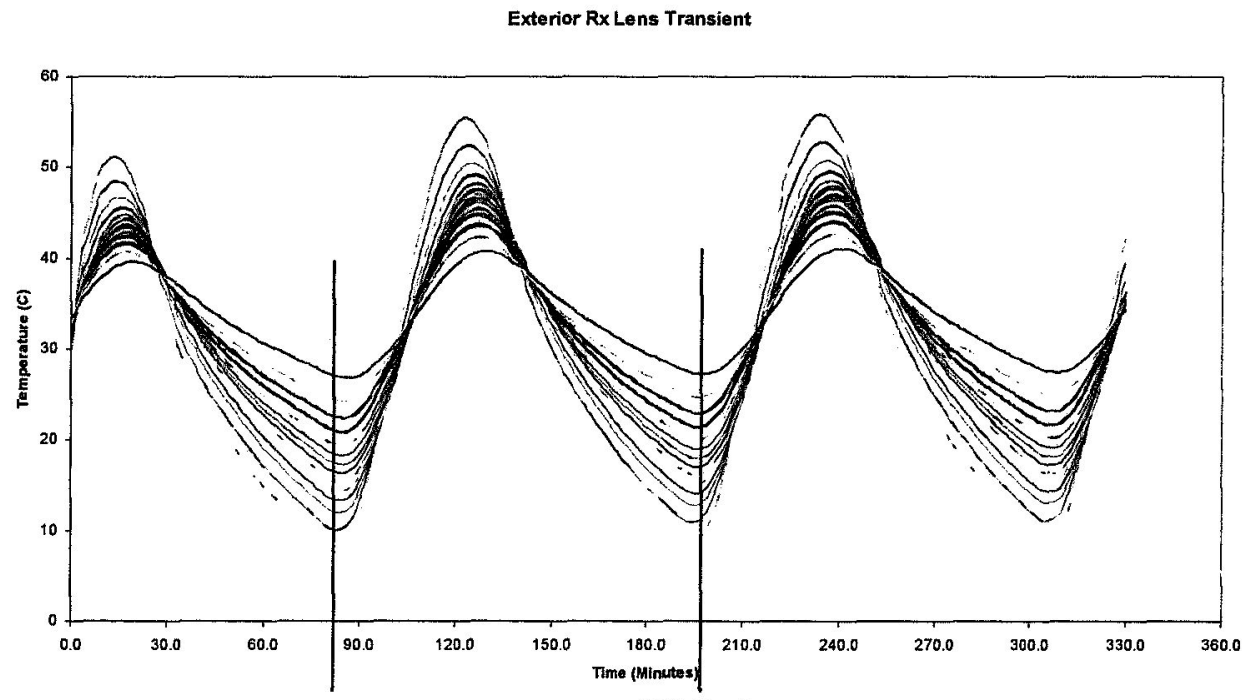

$81.2 \mathrm{~min}$

$195.0 \mathrm{~min}$

Fig. 9. Beta 0 nodal temperatures over three full lunar orbits 
Mapping the temperatures from the thermal model to the structural FEM was accomplished by applying seed temperatures and then running NASTRAN thermal analyzer to interpolate the temperatures on the remainder of the nodes. The seed temperatures were created by applying nodal temperatures to groups of nodes created in FEMAP that corresponded to the locations of the thermal model nodes. To minimize hand mapping in all 21 thermal load cases, a sensitivity analysis was completed to determine the required temperature seeding density. Since stress results were the early focus of the lens analysis, maximum stresses were used as the criteria in the sensitivity analysis. To verify each temperature map, a contour plot of nodal temperatures output by the NASTRAN thermal analyzer was compared to a contour plot of temperatures on the thermal model.

\subsubsection{Point Loads}

A preload of $800 \mathrm{~N}(180 \mathrm{lbs})$ is applied to the lens via a titanium ring flexure with six pads 60 degrees apart. The flexure is shown in Figure 2. For the point load analysis, a line contact was assumed at the edge of flexure pad closest to the center of the lens. Point loads of $66.7 \mathrm{~N}(15 \mathrm{lbs})$ were applied in the $-Z$ direction at two nodes nearest flexure pad corners, as shown in Figure 10.

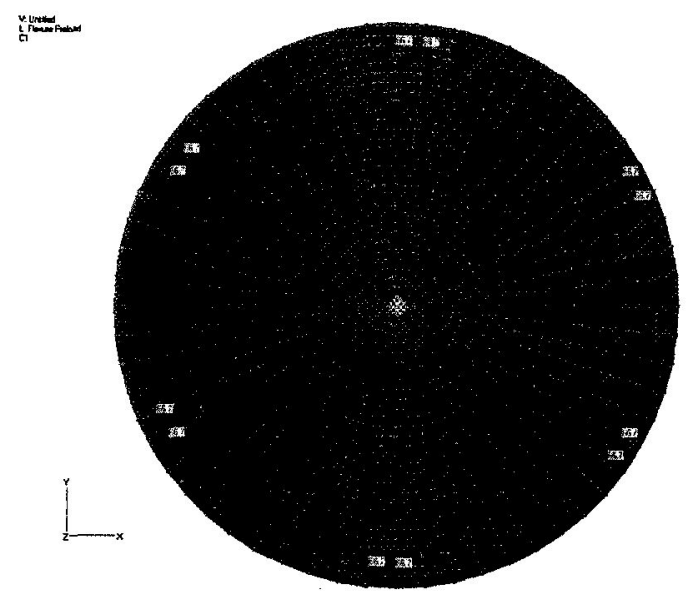

Fig. 10. Applied Preload

\section{ANALYSIS RESULTS}

The first round of analysis combined the thermal and point loads in a single analysis run. When resulting tensile stresses were above the $6.9 \mathrm{MPa}$ allowable, the point loads and thermal loads were run separately to determine the cause of the high stresses.

\subsection{Stress Analysis}

\subsubsection{Point Loads}

The preload induces compression at the six pad locations, as expected. The minimum principal stress is $-6.34 \mathrm{MPa}$, less than the $34.5 \mathrm{MPa}$ compression allowable. A plot of the principal stress is shown below in Figure 11.

\subsubsection{Steady State Cold Temperature Case}

Early structural analysis was based on thermal model results that gave temperatures at only the top and bottom surfaces of the lens. An abrupt gradient in the middle of the glass caused internal stresses, as well as bending of the optic, which resulted in a maximum tensile stress of $7.2 \mathrm{MPa}$, above the $6.9 \mathrm{MPa}$ allowable. A contour plot of maximum stress is shown in Figure 12.

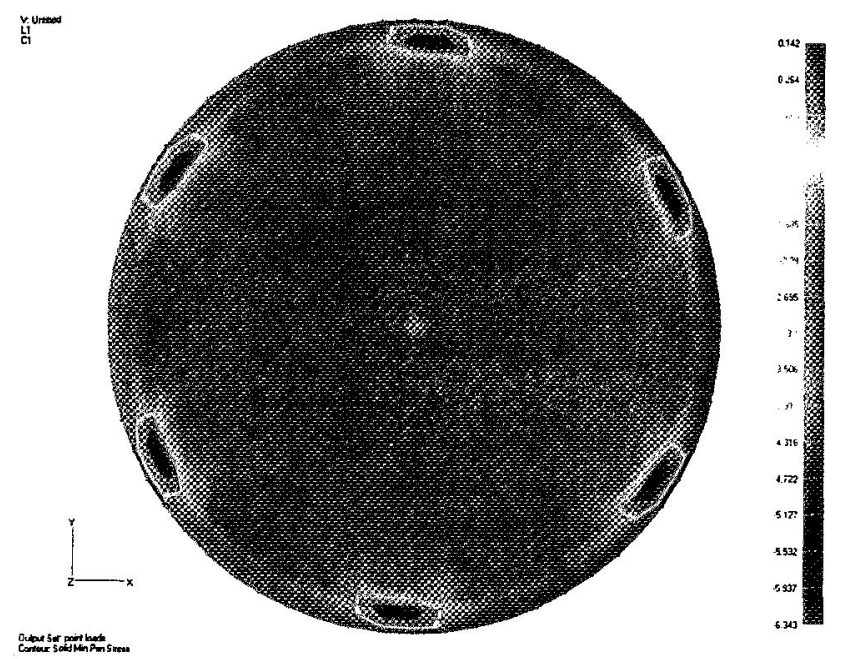

Fig. 11. Contour plot of principal stress (units in MPa) 


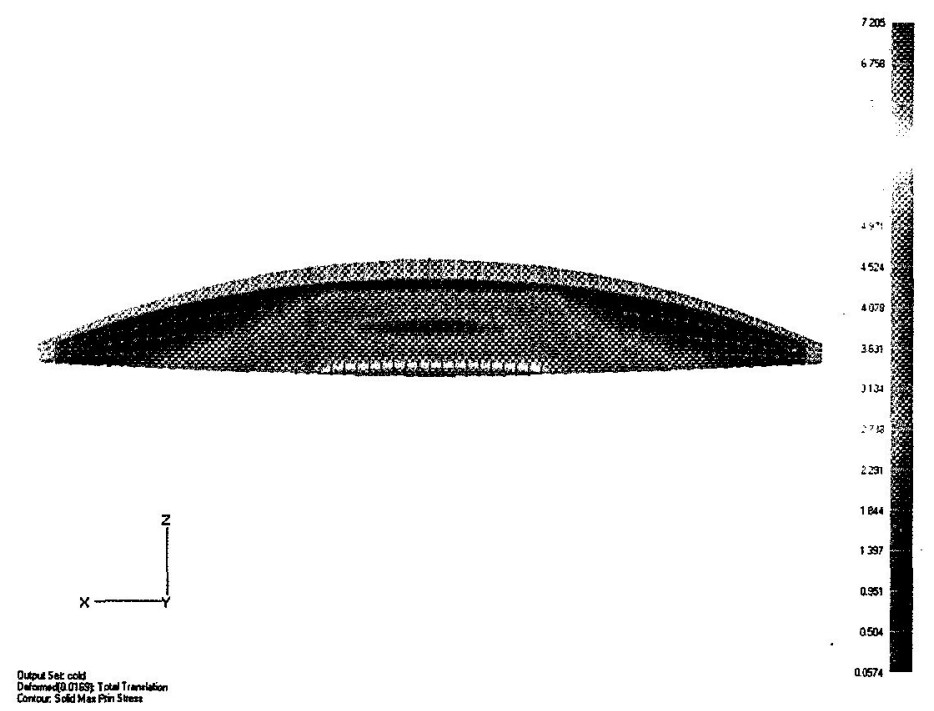

Fig. 12. Contour plot of maximum principal stress (units in MPa) showing lens deformation. Undeformed lens is in gray.

The thermal model was updated with a finer mesh to give a more even temperature distribution through the thickness of the glass. This temperature case has a radial gradient of 24.5 degrees $\mathrm{C}$ - from -24 degrees at the center of the lens to 0.5 degrees at the edges - and an axial gradient of 10 degrees $C$. The plot of applied temperatures for the cold steady state case is shown in Figures 13 and 14. With this updated temperature distribution, lower stress levels of $5.2 \mathrm{MPa}$ in tension and 8.3 $\mathrm{MPa}$ in compression are reported below. The tensile stress is primarily due to bending of the optic and occurs at the bottom surface of the lens, while the compressive stress is internal to the lens, as shown in Figures 15 through 18.

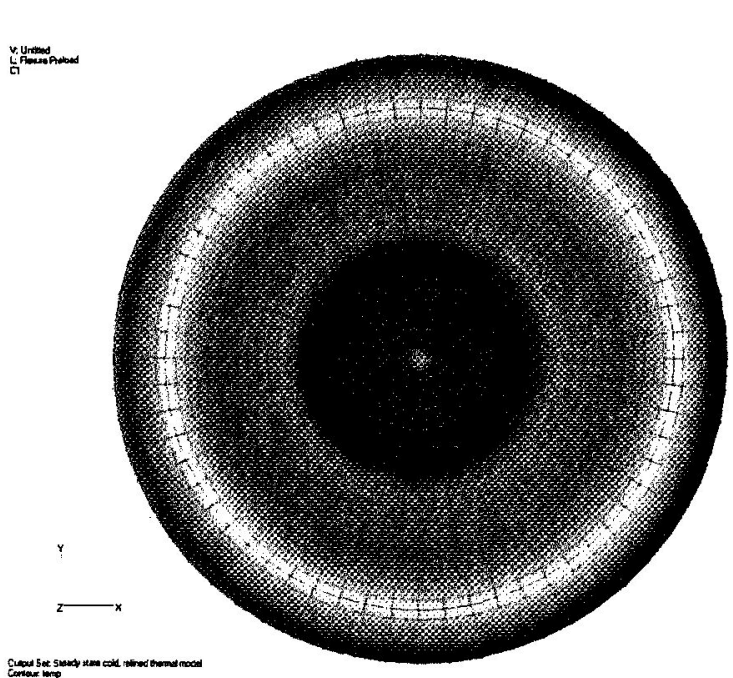

Fig. 13. Contour plot of cold steady state case applied temperatures, top view (units in degrees C)



Fig. 14. Contour plot of cold steady state case applied temperatures, cut away side view (units in degrees $\mathrm{C}$ ) 
Tensile Stress:

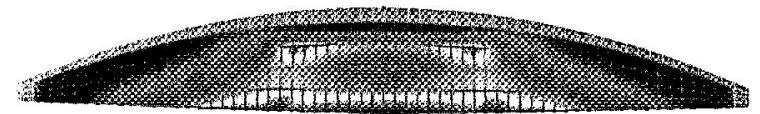

${ }^{818}{ }^{188}$
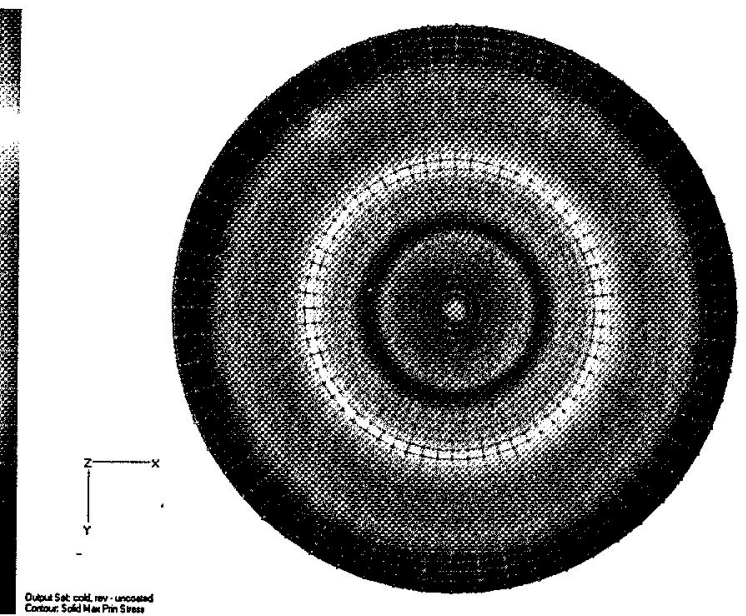

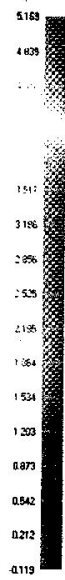

cosits

Fig. 15. Contour plot of maximum principle stress showing lens deformation, cut away side view (units in $\mathrm{MPa}$ )

Fig. 16. Contour plot of maximum principle stress, bottom view (units in $\mathrm{MPa}$ ). Max Principal Stress $=5.2 \mathrm{MPa}$

Compressive Stress:

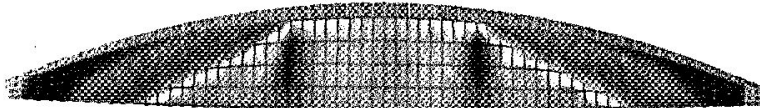

${ }^{1180}$
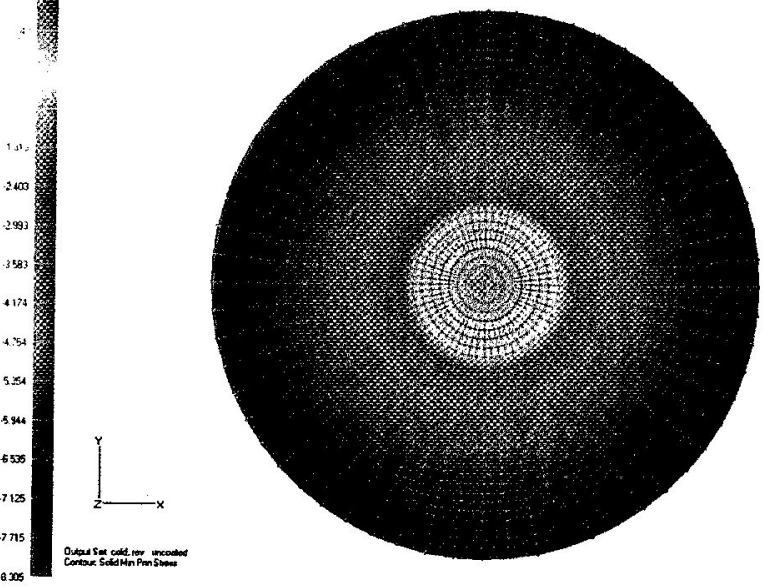

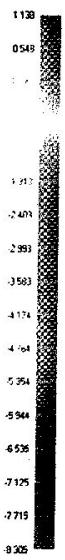

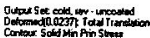

Fig. 17. Contour plot of minimum principle stress showing lens deformation, cut away side view (units in $\mathrm{MPa}$ )

Fig. 18. Contour plot of minimum principle stress, top view (units in MPa). Min Principal Stress $=8.3 \mathrm{MPa}$ 


\subsubsection{Transient Hot Temperature Case}

Twenty temperature cases were mapped to the FEM as described in Section 2.3.1. Sample temperature plots are shown below for the 105.9 minute timestep.

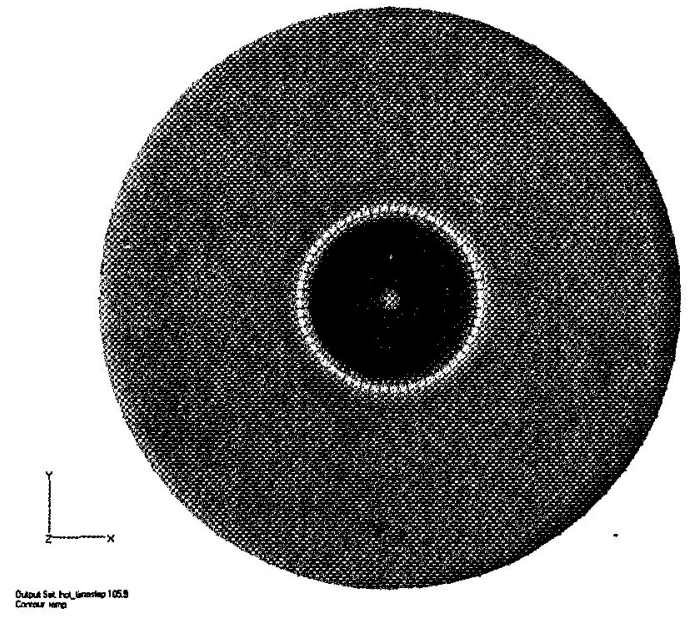

Fig. 19. Contour plot of 105.9 min timestep applied temperatures, top view (units in degrees C)

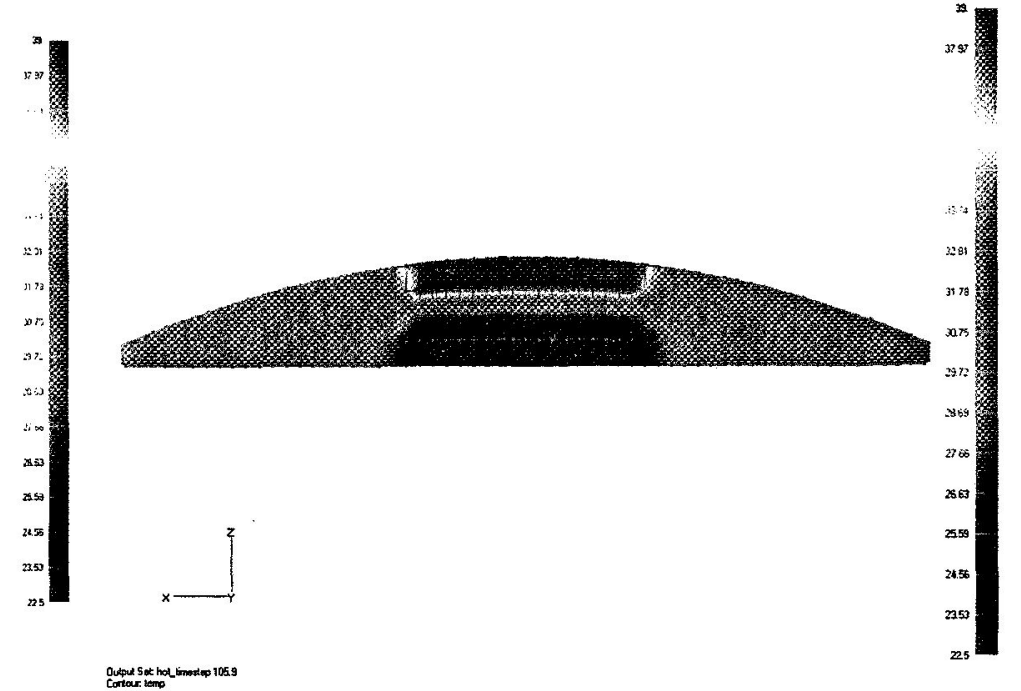

Fig. 20. Contour plot of $105.9 \mathrm{~min}$ timestep applied temperatures, cut away side view (units in degrees $\mathrm{C}$ )

In this transient case, the lens cycles through gradients that produce downward bending similar to the cold steady state case and gradients that produce upward bending. Figure 21 shows the stress contour plot for a downward bending case at the 81.2 minute timestep. An upward bending case, at timestep 105.9 minutes, is shown in Figure 22.

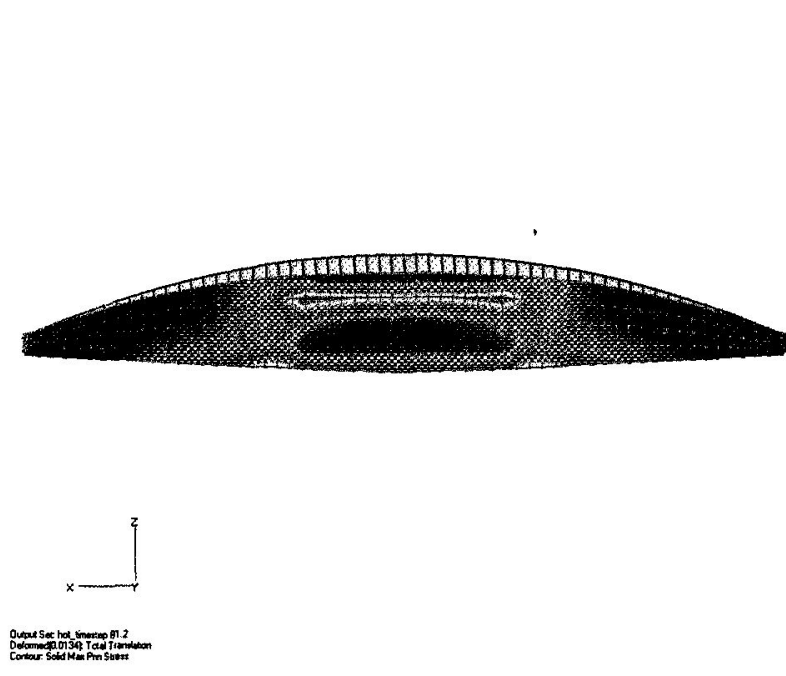

Fig. 21. Timestep 81.2 min. Contour plot of maximum principle stress showing lens deformation, cut away side view (units in MPa)
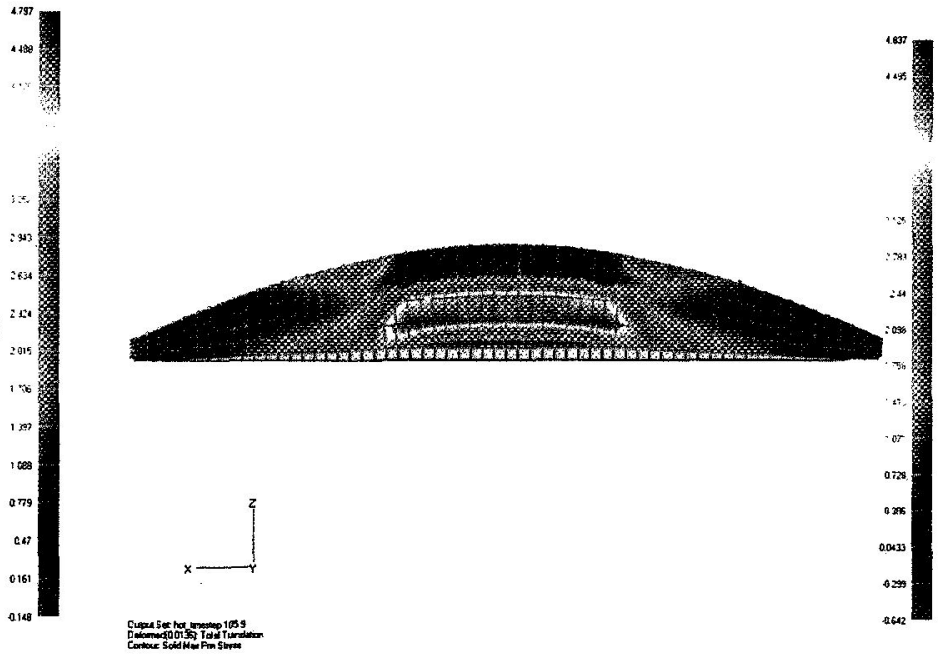

Fig. 22. Timestep 105.9 min. Contour plot of maximum principle stress showing lens deformation, cut away side view (units in $\mathrm{MPa}$ ) 
Results for the 20 temperature cases in the hot transient thermal environment are shown in Table 2. The four cases highlighted in yellow - timesteps $81.2,113.7,120.4$, and 158.8 minutes - were initially chosen to bound the problem because these cases had the largest positive and negative radial and axial gradients. Surprisingly, timestep $105.9 \mathrm{~min}$ yielded the highest tensile stress, $4.8 \mathrm{MPa}$. All stresses were below the allowables.

Table 2

\begin{tabular}{|c|c|c|c|c|c|c|c|}
\hline Hot Cases & $\begin{array}{c}\text { Timestep } \\
\text { (min) }\end{array}$ & $\begin{array}{c}\text { Max Radial } \\
\text { Gradient (deg C) } \\
\end{array}$ & $\begin{array}{c}\text { Max Axial } \\
\text { Gradient (deg C) } \\
\end{array}$ & $\begin{array}{c}\text { Max Compressive } \\
\text { Stress (MPa) }\end{array}$ & $\begin{array}{c}\text { Max Compressive } \\
\text { Stress (psi) }\end{array}$ & $\begin{array}{l}\text { Max Tensile } \\
\text { Stress (MPa) } \\
\end{array}$ & $\begin{array}{l}\text { Max Tensile } \\
\text { Stress (psi) }\end{array}$ \\
\hline 1 & 81.2 & 19.40 & -12.27 & -5.349 & -776 & 4.797 & 696 \\
\hline 2 & 87.2 & 17.79 & -9.33 & -5.523 & -801 & 3.75 & 544 \\
\hline 3 & 93.2 & 11.41 & -1.22 & -5.003 & -726 & 3.444 & 499 \\
\hline 4 & 99.2 & 3.08 & 8.03 & -3.93 & -570 & 4.276 & 620 \\
\hline 5 & 105.9 & -6.06 & 16.46 & -5.294 & -768 & 4.837 & 86 \\
\hline 6 & 111.4 & -11.98 & 20.38 & -6.665 & -967 & 4.555 & 661 \\
\hline 7 & 113.7 & -13.71 & 21.00 & -6.838 & -992 & 4.512 & 654 \\
\hline 8 & 120.4 & -15.90 & 19.33 & -6.378 & -925 & 4.506 & 654 \\
\hline 9 & 126.8 & -13.84 & 13.36 & -4.417 & -641 & 3.454 & 501 \\
\hline 10 & 133.6 & -7.72 & 3.80 & -2.012 & -292 & 2.68 & 389 \\
\hline 11 & 139.7 & 0.04 & -5.69 & -2.913 & -422 & 2.023 & 293 \\
\hline 12 & 145.4 & 5.49 & -10.99 & -2.841 & -412 & 3.655 & 530 \\
\hline 13 & 151.7 & 8.71 & -12.75 & -2.797 & -406 & 4.23 & 613 \\
\hline 14 & 158.8 & 11.32 & -13.05 & -3.105 & -450 & 4.415 & 640 \\
\hline 15 & 165.2 & 13.27 & -12.98 & -3.339 & -484 & 4.534 & 658 \\
\hline 16 & 171.6 & 14.96 & -12.84 & -3.514 & -510 & 4.607 & 668 \\
\hline 17 & 178.4 & 16.50 & -12.69 & -3.943 & -572 & 4.705 & 682 \\
\hline 18 & 184.8 & 17.76 & -12.55 & -4.538 & -658 & 4.758 & 690 \\
\hline 19 & 190.1 & 18.65 & -12.45 & -4.951 & -718 & 4.824 & 700 \\
\hline 20 & 1.95 & 18.66 & -11.47 & -5.274 & -765 & 4.488 & 651 \\
\hline
\end{tabular}

\subsection{Distortion Analysis}

Distortions due to the thermal gradients were provided to LOLA's lead optical analyst at each node along the top and bottom surfaces of the lens cross-section. Contour plots below show the lens deformation for the cold operational case and the worst case from the hot transient environment, the 113.7 minute timestep.

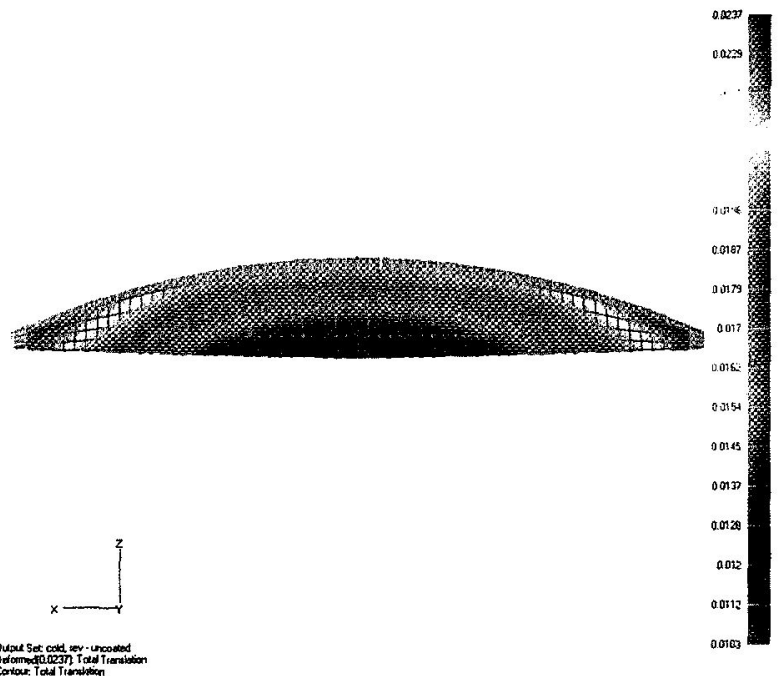

Fig. 23. Cold Operational Case. Deformed view showing contour plot of total translation, cut away side view (units in $\mathrm{mm}$ )

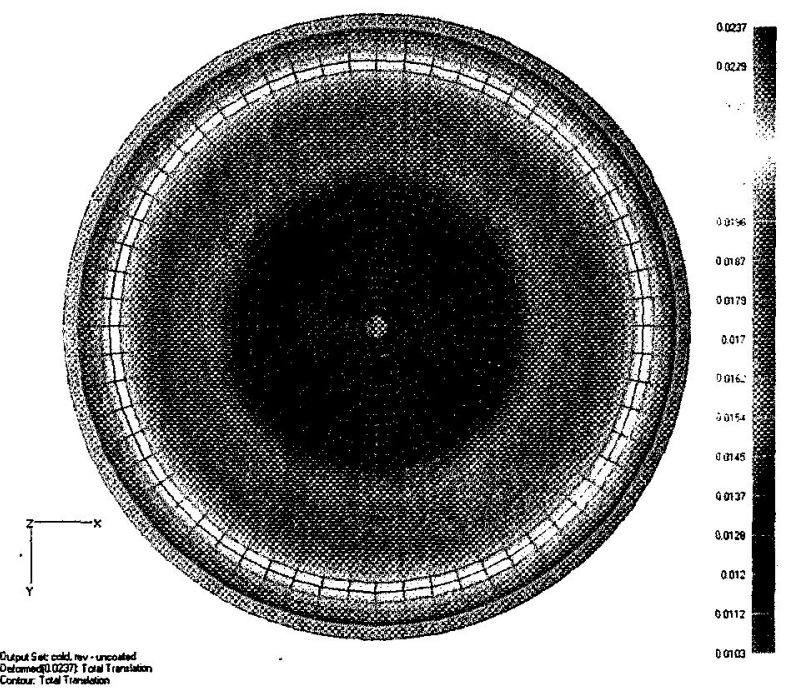

Fig. 24. Cold Operational Case. Deformed view showing contour plot of total translation, bottom view (units in $\mathrm{mm}$ ) 


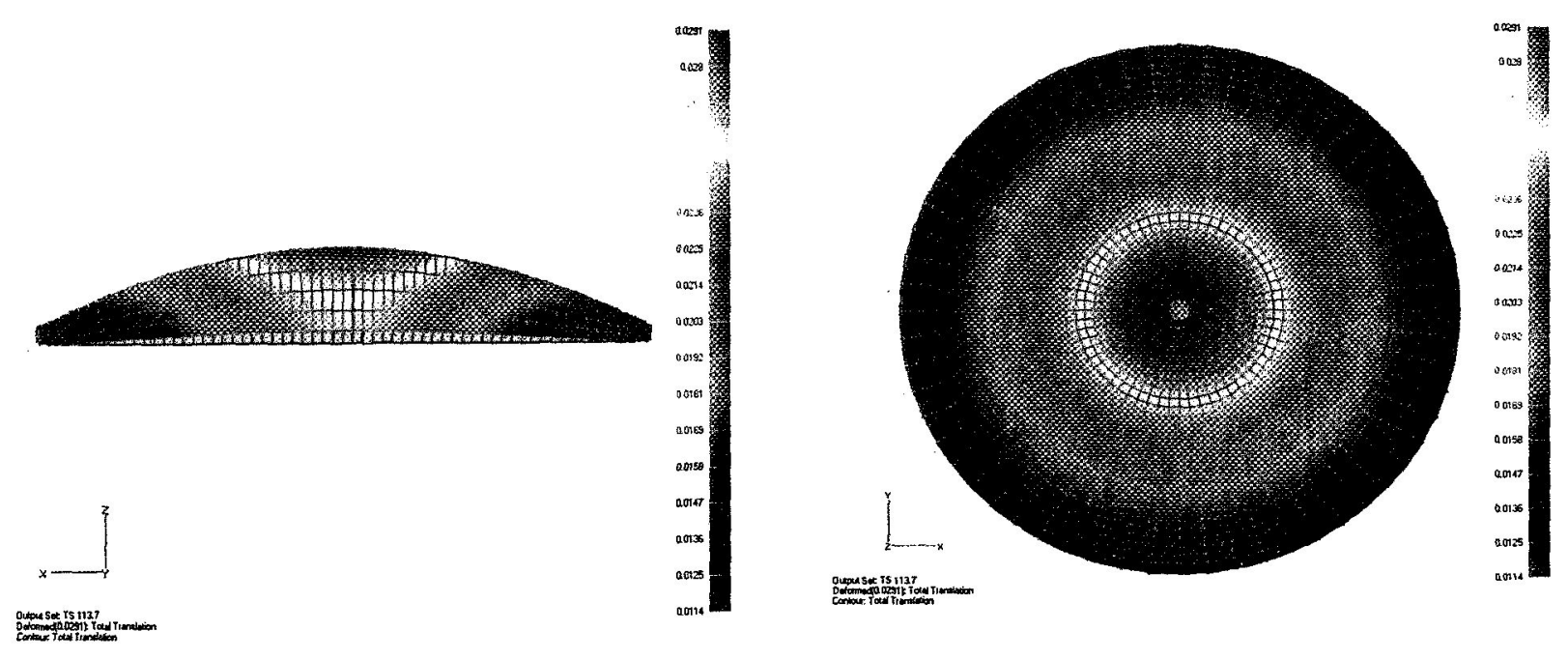

Fig. 25. Hot timestep $113.7 \mathrm{~min}$. Deformed view showing contour plot of total translation, cut away side view (units in $\mathrm{mm}$ )
Fig. 26. Hot timestep $113.7 \mathrm{~min}$. Deformed view showing contour plot of total translation, bottom view (units in $\mathrm{mm}$ )

The maximum displacement in all temperature cases occurs in the 113.7 minute timestep of the hot transient case. The maximum total translation of 29.1 microns is primarily translation in the $Z$ direction. The maximum displacement in the cold steady state case is 23.7 microns, of which 19.8 microns is motion in the $Z$ direction. The hot 113.7 minute timestep case produced the most bending, while the cold steady state case exhibited bending with radial contraction.

Raytrace results by the optical analyst showed that these deformations would lead to significant image blur. Thus, the thermal gradients in the lens needed to be reduced from an operational perspective.

\subsection{Design Change}

In order to reduce the thermal gradients in the lens, a variety of design changes were studied by the author and LOLA's lead thermal analyst. These design changes included lens coatings, baffling, and different lens materials. The design team decided to use sapphire for the receiver lens material because thermal analysis showed significantly smaller thermal gradients with sapphire than with BK7 glass. With operational thermal gradients of less than two degrees $\mathrm{C}$, and higher strength allowables, sapphire was the best lens material choice. In addition, MLA's receiver telescope used a sapphire objective lens, bringing the LOLA design in line with that of its predecessor.

\section{CONCLUSIONS}

Thermal analysis of the BK7 objective lens showed large on-orbit thermal gradients, on the order of 25 degrees $\mathrm{C}$ radial and 10 degrees $C$ through the thickness of the lens. These thermal gradients bend the lens, which leads to lens internal stresses and de-focusing of the receiver image. Initial analysis showed significant receiver de-focus and lens stress. In addition, optical analysis showed that the change in index of refraction with internal temperature further contributes to receiver de-focus. While further analysis showed lens internal stresses to be below the tensile allowable, optical performance could be greatly improved by using a sapphire lens instead. This change brought the design back to the original MLA-based receiver design to mitigate risk. Sapphire operates closer to the 20 degree C integration temperature, has no on-orbit gradients, and is a higher strength material. The improvement to optical performance outweighed the additional mass required for the sapphire lens.

Bounding the hot transient case proved to be a challenge, as surprising stress results came from the 20 timesteps chosen in the second orbit. The first four timesteps chosen, those with the largest positive and negative radial and axial gradients, did include the case with maximum predicted distortion. However, the largest stress prediction occurred in a 
case with an axial gradient of 16.5 degrees $C$, less than the 21 degree $C$ maximum, and a radial gradient of -6.1 degrees, less than half the most negative radial gradient of 15.9 degrees. Two timesteps, 190.1 and 105.9 minutes, had maximum stresses higher than that of the initial bounding cases. This illustrates the challenge of identifying the worst case thermal loads in transient cases with many timesteps.

\section{ACKNOWLEDGEMENTS}

The author gratefully acknowledges Carlton Peters and Luis Ramos-Izquirdo for their work in the thermal and optical portions of the lens analysis. Thanks also to Steve Schmidt and Walter Thomas for mechanical support.

\section{REFERENCES}

1. G. Chin, S. Brylow, M. Foote, J. Garvin, J. Kasper, J. Keller, M. Litvak, I. Mitrofanov, D. Paige, K. Raney, M. Robinson, A. Sanin, D. Smith, H. Spence, P. Spudis, S. Stern, M. Zuber, "Lunar Reconnaissance Orbiter Overview: The Instrument Suite and Mission," Space Science Reviews, May 2007.

2. C. Peters, "Thermal Subsystem Final Design," CDR Package, July 2006.

3. L. Ramos-Izquierdo, "Optics Subsystem Final Design," CDR Package, July 2006.

4. H. Riris, X. Sun, J. Cavanaugh, G. Jackson, L. Ramos-Izquierdo, D. Smith, and M. Zuber, "The Lunar Orbiter Laser Altimeter (LOLA) on NASA's Lunar Reconnaissance Orbiter (LRO) Mission," Proc. of SPIE Vol. 6555, Sensors and Systems for Space Applications, May 2007.

5. S. Schmidt, S. Wall, E. Matzinger, D. Brown, "LOLA Mechanical Peer Review II," August 2006. 